\title{
Auswirkungen der globalen Klimaerwärmung auf Wachstum und Winterhärte von Bäumen in Nordwest-Russland
}

\author{
Gennady A. Firsov \& Inna V. Fadeyeva
}

\begin{abstract}
The warming of the climate at Saint-Petersburg (NW Russia) has clearly increased since summer 1988. This allowed to cultivate successfully outdoors many more warm loving trees of southern origin than it was possible before.
\end{abstract}

\section{Zusammenfassung}

Die Klimaerwärmung wird in St. Petersburg (Nordwest-Russland) seit Sommer 1988 deutlich. Dadurch lassen sich nun im Freien viel mehr wärmeliebende Bäume südlicher Breiten als früher kultivieren.

\section{Egbert Wolf}

Im Oktober 2010 fand in St. Petersburg an der Forsttechnischen Akademie die Tagung „Dendrologie zu Beginn des 21. Jahrhunderts" statt. Die Tagung sollte an Egbert Wolf (18601931) erinnern. Der bekannte deutsch-russische Dendrologe, Systematiker und Gärtner hätte im letzten Jahr seinen 150. Geburtstag gefeiert.

Wolf wurde am 5. September 1860 in Berlin geboren. Er besuchte dort von 1871-1876 das Realgymnasium, später (1876-1879) die Gärtnerschule am Botanischen Garten Berlin. Im Jahr 1882 ging er auf Einladung von E. L. REGEL, dem Direktor des botanischen Gartens in St. Petersburg, nach Russland. Bereits am 4. September 1886 wurde er zum leitenden Gärtner des russischen Forstinstitutes (heute FTA) ernannt. Hiermit begannen seine erfolgreichen Aktivitäten im Bereich der Dendrologie.

\section{WOLFS dendrologische Untersuchungen}

Als herausragender Gehölzkenner untersuchte Wolf die Einführungsgeschichte sowie Winterhärte und Kulturbedingungen einer großen Zahl eingeführter Gehölze (Wolf 1917). Viele von ihnen wurden seit dieser Zeit nicht wieder

Abb. 1 (oben): Gennady Firsov neben einer Heckenkirsche (Lonicera tolmatchevii).

Abb. 2 (unten): InNA V. FAdeyeva bewundert eine Japanische Kaiser-Eiche, die nun auch in St. Petersburg im Freien kultivierbar ist.
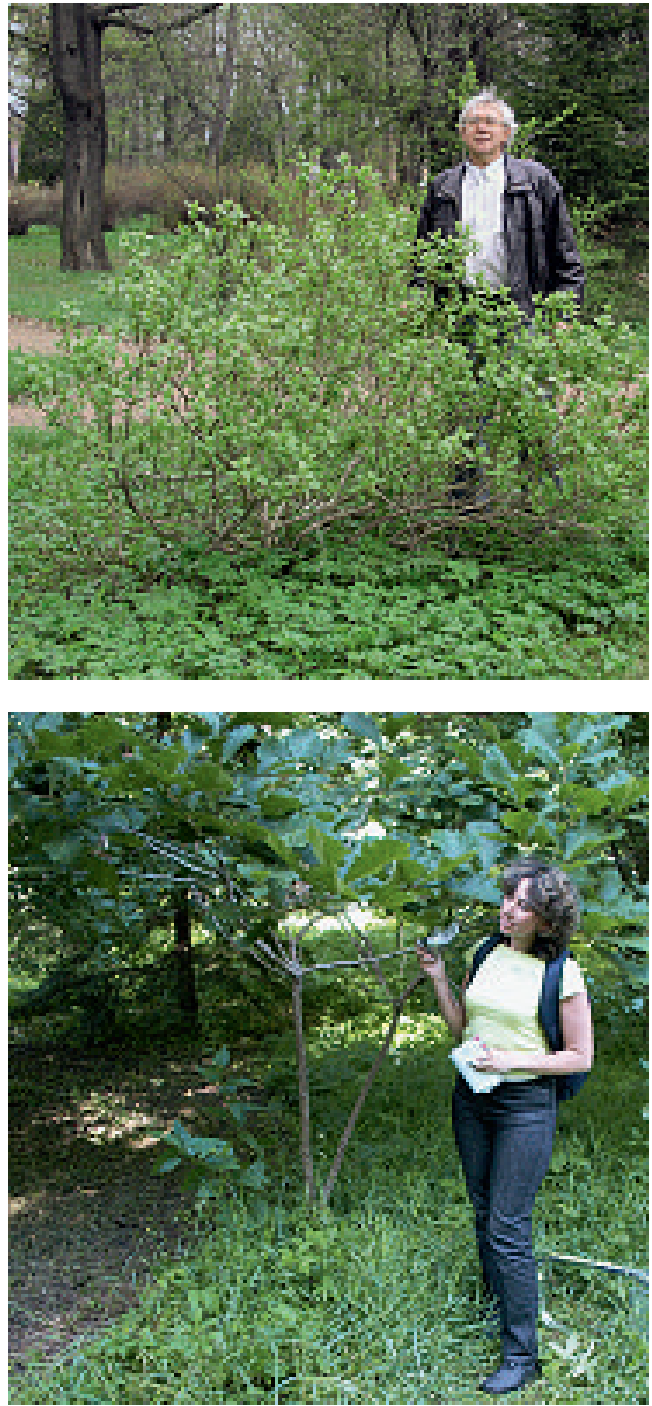


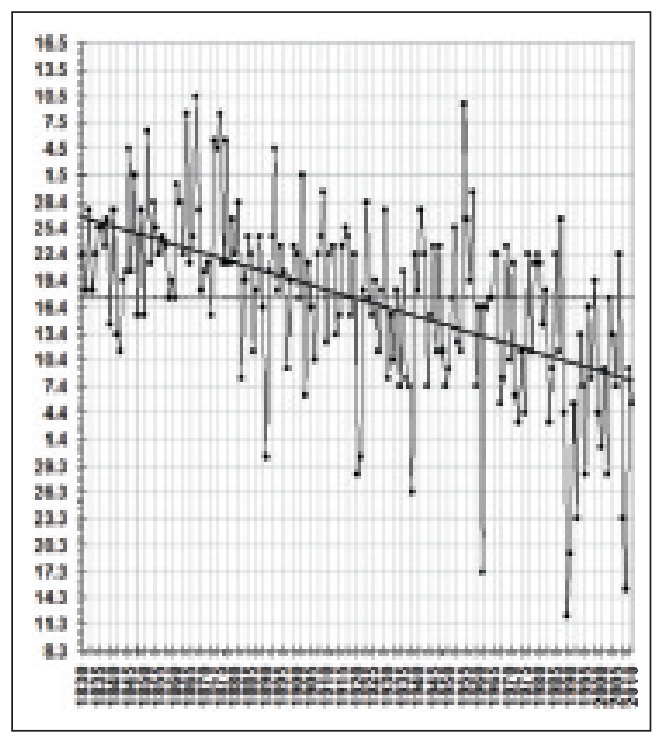

gesichtet, sodass etliche heutige Kulturanleitungen noch immer auf Wolfs Daten basieren und nicht den aktuell veränderten klimatischen Bedingungen angepasst sind. Für seine Beobachtungen legte Wolf in der Forsttechnischen Akademie eine umfangreiche Gehölzsammlung an. Einige der von ihm gepflanzten Bäume existieren noch heute, darunter Acer mandshuricum und Pinus peuce. Wolf starb 1931. Er wurde im Park unter Bäumen beerdigt, die viele Jahre zuvor von ihm gepflanzt worden waren.

Wolf hatte erkannt, dass ein wesentlicher Aspekt für die Freiland-Kultur von Bäumen und Sträuchern ihr Grad der Winterhärte und Frosttoleranz ist. Seine Winterhärte-Skala aus dem Jahr 1917 ist eine der bekanntesten und wird noch heute neben der von Heinze \& Schreiber (1984) von vielen Dendrologen zu Rate gezogen. Er betonte in seinen Publikationen, dass das Klima in St. Petersburg, einer Stadt an der Grenze zum dunklen Norden, hart und streng sei (Wolf 1929) und sich dort deshalb viele eingeführte Gehölze an der Grenze ihrer Wachstumsmöglichkeiten befinden. St. Petersburg liegt auf einer nördlichen Breite von $60^{\circ}$. Die Winter sind dort dementsprechend deutlich strenger als in Berlin, Frankfurt und Hamburg. Der Winter dauert in St. Petersburg rund vier Monate. Die niedrigste Temperatur, die dort jemals gemessen wurde, betrug am 17. Januar $1940-35,6^{\circ} \mathrm{C}$.

\section{Klimawandel}

Im Vergleich mit dem Klima zu Lebzeiten von Egbert Wolf hat sich das aktuelle Klima in St. Petersburg unübersehbar verändert und ist deutlich milder geworden. Die 2. Hälfte des 20 . Jahrhunderts wurde von Klimatologen als eine Periode des modernen Klimas bezeichnet. Die Jahresdurchschnittstemperatur lag in diesem Zeitraum in St. Petersburg bei $4,3{ }^{\circ} \mathrm{C}$ (Pokrovskaya \& Bychkova 1967, Shver et al. 1982). Es kam dann zu einer Erhöhung um $1,5^{\circ} \mathrm{C}$, so dass für den Zeitraum von 1980-2009 eine Durchschnittstemperatur von $5,8^{\circ} \mathrm{C}$ angegeben werden kann. Diese zunächst gering erscheinende Erhöhung hat nicht unerhebliche Auswirkungen auf die Länge der Vegetationsperiode, die sich in St. Petersburg um beachtliche 11,7 $\pm 0,97$ Tage erhöht hat (FADEyeva \& Firsov 2010).

Eine spürbare Erwärmung des Klimas in St. Petersburg ist seit dem Sommer 1988 festzustellen. Das darauffolgende Jahr 1989 war mit einem Durchschnittswert von $7,6^{\circ} \mathrm{C}$ das wärmste Jahr seit Beginn der Wetteraufzeichnungen im Jahre 1743. Es gibt Prognosen, dass solche

Abb. 3 (oben): Beginn der Blütezeit von Alnus incana, ein Indikator für klimatische Schwankungen. Nach extrem kalten Wintern vor einigen Jahrzehnten erschienen die Kätzchen erst im April oder Mai (27. 4. 1941; 22. 4. 1942; 26. 4. 1987 und 9. 5. 1955). In extrem warmen Jahren konnten sie dagegen schon im März oder Anfang April beobachtet werden (17.3. 1961; 3.4. 1973; 4.4.1999 und 12.3. 1989). Aufblühzeit nach dem 19.4: Später Kalt-Zyklus; Aufblühzeit vor dem 14.4.: Früher Warm-Zyklus. In Mitteleuropa wird meist die ähnliche Schwarz-Erle (Alnus glutinosa) für phänologische Untersuchungen herangezogen. Das Öffnen ihrer Kätzchen (in Mitteleuropa durchschnittlich zwischen Ende Februar und Anfang März) kennzeichnet den Vorfrühling.

Abb. 4 (Seite 45 oben): Japanische Rotkiefer (Pinus densiflora). 
Anomalien in Zukunft häufiger werden könnten (Komarova \& Firsov 1995). Ein Hinweis auf diese Tendenz könnte sein, dass es in der Mitte des 20. Jahrhunderts noch alle 8-13 Jahre extrem kalte Winter gegeben hat, die seit 1986/87 ausgeblieben sind.

\section{Veränderte Winterhärte}

Dementsprechend hat sich für einige Arten der limitierende Einfluss des Frostes vermindert. In Tabelle 1 sind Winterhärte und Fähigkeit zur Reproduktion für acht Arten während unterschiedlicher Zeiträume zusammengefasst.

Einige Arten, die früher wegen ihrer zu geringen Winterhärte in St. Petersburg nicht als kulturfähig galten, sind inzwischen für die Kultur interessant und vielversprechend geworden. Viele dieser Arten überstehen nun den Winter im Freien, entwickeln Früchte mit keimfähigen Samen oder sähen sich sogar selbst aus. Allerdings sollte berücksichtigt werden, dass sporadische, wenn auch vielleicht seltener auftretende extrem kalte Winter zu Rückschlägen führen können, wie es auch die letzten kalten Winter in Mitteleuropa bewiesen haben.

Klimatologen und Dendrologen interessieren sich aktuell sehr für Zusammenhänge zwischen Phänologie und Klimaveränderungen. Die Be-

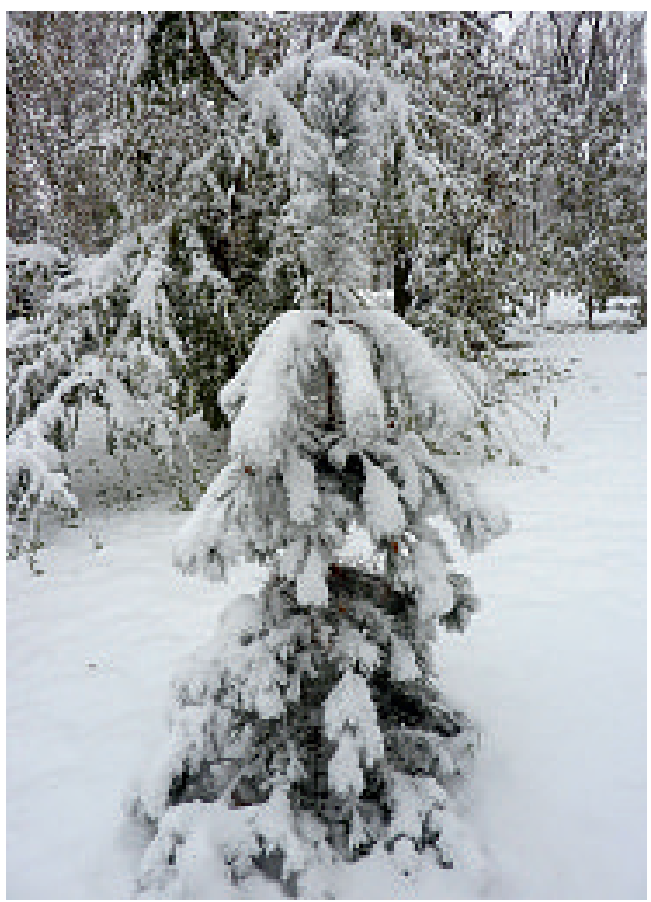

obachtung des Wachstums, der Laub-, Blütenund Fruchtentwicklung von Pflanzen ist eine gute Methode, meteorologische Beobachtungen zu ergänzen. Klimatologische Veränderungen spiegeln sich in Änderungen der Phänologie wi-

\begin{tabular}{|l|l|l|l|}
\hline \multirow{2}{*}{ Art } & \multicolumn{3}{l|}{ Winterhärte und Reproduktion } \\
\cline { 2 - 5 } & I886-19I6 & I948-I977 & I978-2007 \\
\hline Abies holophylla Maxim. & II, Veg & I-II, Fr & I, Fr \\
\hline Abies nordmanniana (Stev.) Spach & III-IV, Veg & III-IV, Veg & III-IV, Fr \\
\hline Acer barbinerve Maxim. & II, Fr & I, Fr, S & I, Fr, S \\
\hline Acer cissifolium (Sieb. et Zucc.) C. KocH & IV, Veg & III-IV, Veg & II-III, Fr \\
\hline Acer japonicum Thunb. & V & III-IV, Veg & III-IV, Fr \\
\hline Acer mandshuricum Maxim. & II, Fr & I, Fr, S & I, Fr, S \\
\hline Acer palmatum Thunb. & V & III-IV, Veg & III-IV, Fr \\
\hline Acer rubrum L. & I, Bl & I, Fr & I, Fr \\
\hline
\end{tabular}

Tabelle 1: Anstieg der Winterhärte von Bäumen in St. Petersburg (1886-2007). 1886-1916: Zeit, aus der WolfS Ergebnisse stammen, bevor er seine Ergebnisse 1917 veröffentlichte; Winterhärtegrade: I - ziemlich hart; II - vergleichsweise hart; III - vergleichsweise nicht hart; IV - nicht hart; V - absolut nicht hart, bereits den ersten Winter nicht überlebend. Fr - Früchte, Bl - Blüten, Veg - nur vegetativ, S - Selbstaussaat. 


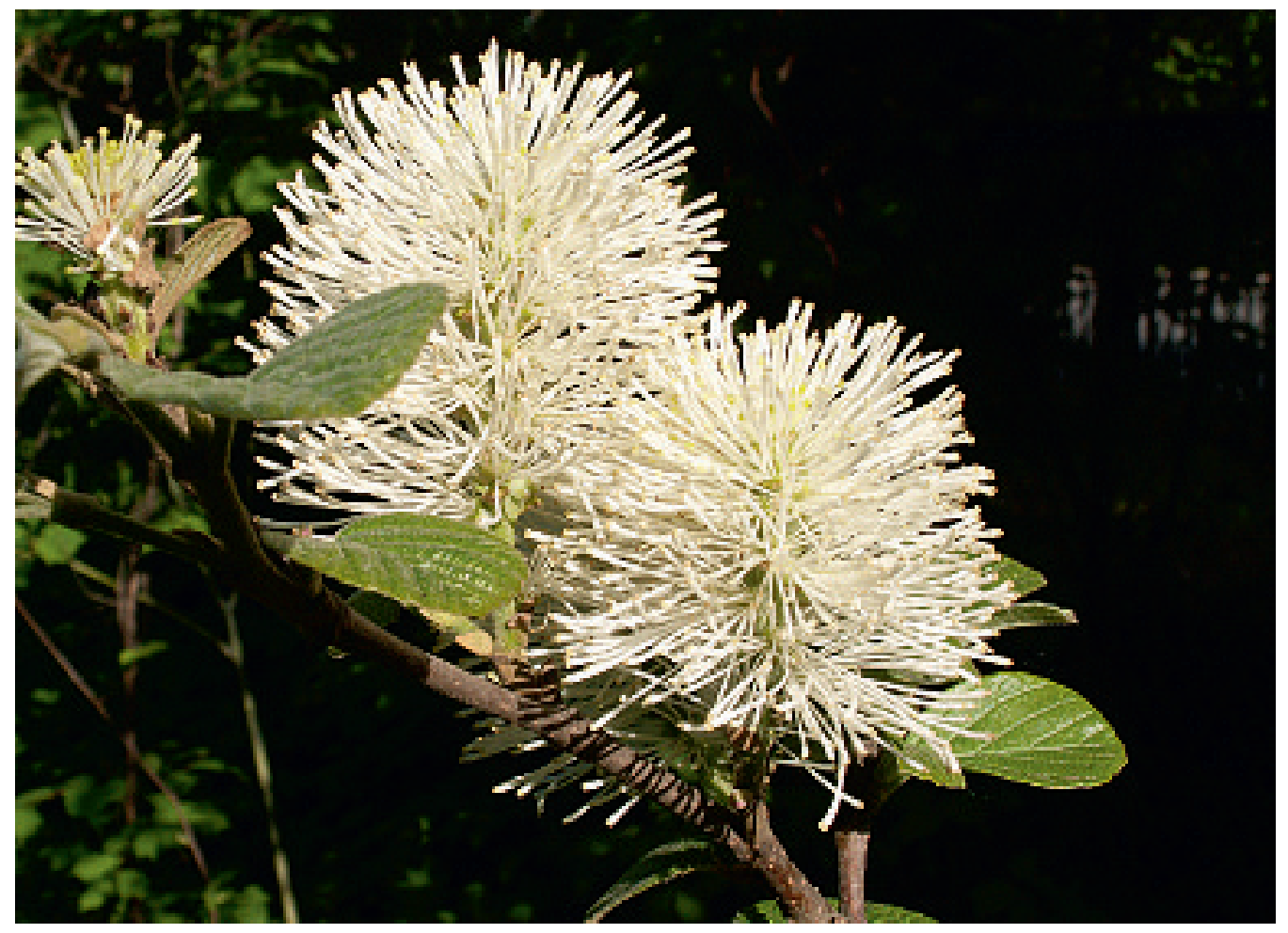

der. Nikolai Buligin befasst sich in Russland intensiv mit dieser Thematik. Für derartige Untersuchungen sind möglichst kontinuierliche, langjährige phänologische Aufzeichnungen unerlässlich. In der Forsttechnischen Akademie St. Petersburg wurden phänologische Daten zusammengetragen, die bis auf das Jahr 1829 zurückgehen. Sowohl die meteorologischen als auch die phänologischen Daten sind zyklischen Schwankungen unterworfen.

Trotz der allgemeinen Tendenz zur Erwärmung wechseln sich noch immer sogenannte frühe Warm- und späte Kalt-Zyklen (mit noch spät im Frühjahr auftretenden Spätfrösten) ab. In solchen Jahren zeigen gleiche Arten ganz unterschiedliches Verhalten in ihrem Biorhythmus, ihrer Winterhärte sowie ihrer Fähigkeit zur Reproduktion. Diese zyklisch auftretenden Schwankungen der Phänologie wurden als bioklimatische Zyklen bezeichnet (FADEYeVA et al. 2009). Eine gute Indikator-Art für besonders warme bzw. kalte Jahre ist die in Russland heimische Grau-Erle (Alnus incana).
Das Aufblühen der männlichen Kätzchen der Grau-Erle ist Zeichen des Frühlingsbeginns und markiert allgemein den Start der Vegetationsperiode. Neben heimischen Arten werden aber auch exotische untersucht. Bei gut angepassten Arten wirken sich meteorologische Schwankungen eher geringfügig auf die Phänologie aus. Die stärksten Veränderungen und Schwankungen ihrer Phänologie zeigen Arten, die im Untersuchungsgebiet gerade noch kultiviert werden können und sich an ihrer Grenze der Winterhärte befinden. Manche wärmeliebenden Bäume und Sträucher konnten bisher nur in Jahren mit relativ kurzen bzw. milden Wintern kultiviert werden. Im Zuge der Klimaerwärmung werden sich die meteorologischen und phänologischen Zyklen verändern mit ei-

Abb. 5 (Seite 46): Federbuschstrauch (Fothergilla major).

Abb. 6 (Seite 47 oben): Hickory (Carya ovata).

Abb. 7 (Seite 47 unten): Persischer Flieder

(Syringa persica). 
nem früheren Beginn der Vegetationszeit und Chancen für wärmeliebendere Arten. Alnus incana blüht tendenziell immer früher im Jahr.

Die allgemeine Erwärmung ist für Gärtner Herausforderung und Chance zugleich, denn sie können nun ein breiteres Spektrum wärmeliebenderer Arten kultivieren (Grismshaw \& BAyton 2009), wie es auch in St. Petersburg bereits praktiziert wird (FIrsov et al. 2010). Einige Arten, die früher nicht als winterhart galten, sich heute aber in St. Petersburg kultivieren lassen, sind auf den Abbildungen 1, 2 und 4-10 dargestellt. Mit der Erwärmung kann es aber auch zu nachteiligen Auswirkungen auf Gehölze kommen, wie der extrem warme Winter 2006/7 zeigte. Der Zeitraum winterlicher Witterung war mit 41 Tagen (20. 1.-2. 3.) relativ kurz. Der Dezember war mit einer durchschnittlichen Temperatur von $+0,8^{\circ} \mathrm{C}$ rekordverdächtig warm. Die herbstliche Witterung hielt etwa 5 Monate lang an. In den letzten 20 Jahren lag die durchschnittliche Monatstemperatur der Monate Dezember bis März zwischen rund $-8{ }^{\circ} \mathrm{C}$ und $-2{ }^{\circ} \mathrm{C}$. Das unnatürlich warme Wetter im Dezember und Anfang Januar rief bei vielen Bäumen einen frühen Entwicklungsstart mit vorgezogener Blütenöffnung hervor. Dieser wurde dann aber durch die später auftretenden Fröste bis $-22^{\circ} \mathrm{C}$ (Firsov et al. 2008) gebremst. Als weiteres Problem trat nach warmen Wintern ein Brüchigwerden einiger Gehölze auf, das auf Fäulnis an der Stammbasis zurückzuführen war. Nach dem extrem heißen Sommer 2010 in allen europäischen Teilen Russlands muss davon ausgegangen werden, dass auch Hitzebeständigkeit (bei in freier Landschaft gepflanzten Gehölzen auch Feuerresistenz) ebenso wie die Frosthärte wichtige limitierende Faktoren für das Gedeihen und die Kultur diverser Gehölze darstellen.

Angepasst an die Klimaerwärmung müssen vermutlich in Zukunft unter Berücksichtigung neuer Schädlinge und Krankheiten veränderte Kulturtechniken und ein anderes Artenspektrum in Landwirtschaft und Zierpflanzenbau berücksichtigt werden. Die kontinuierliche Beobachtung der Vitalität und Phänologie bestimmter Arten ist dabei ein wichtiges Instru-
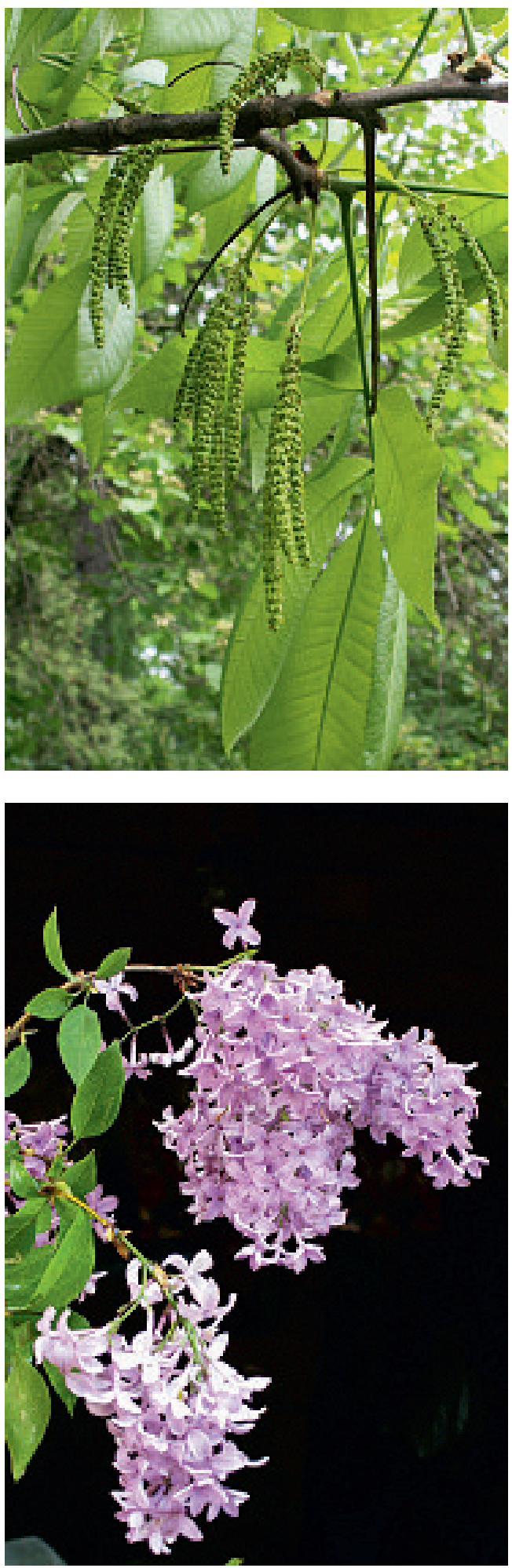

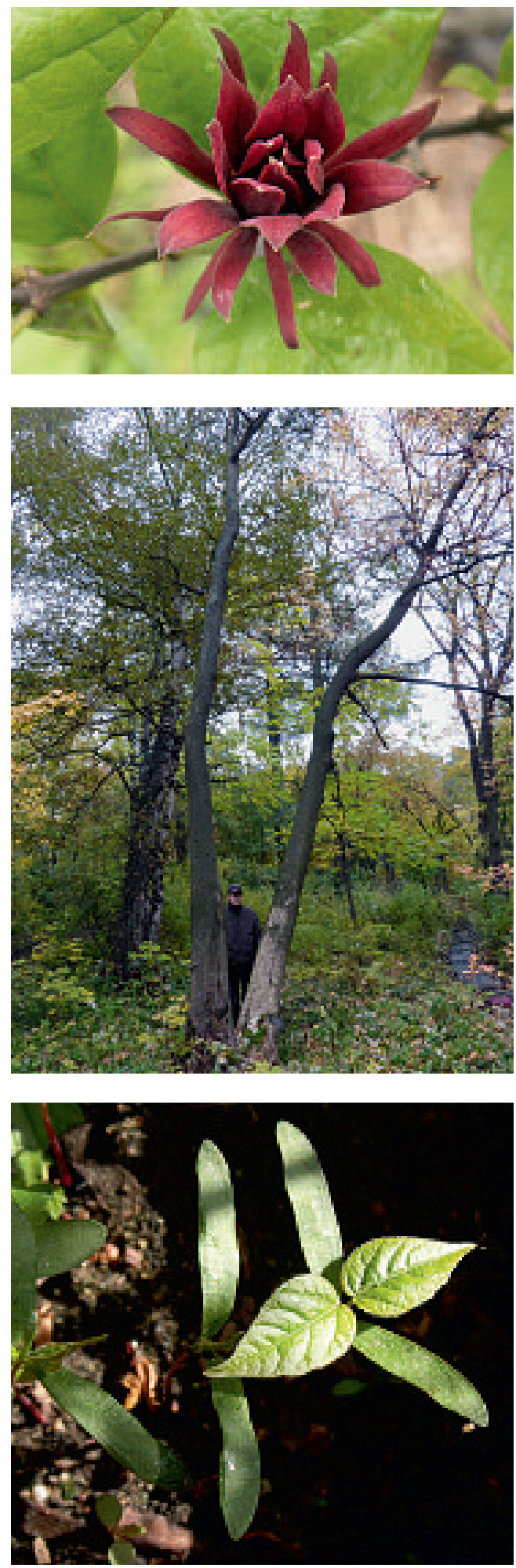

ment, die Veränderungen festzustellen. St. Petersburg ist in Russland für derartige Untersuchungen ein Vorbild, denn hier sind die kontinuierlichsten Messreihen zur russischen Meteorologie und Phänologie zusammengetragen.

\section{Literatur}

Fadeyeva, I.V., Firsov, G. A. \& Buligin N. E. 2009:

Bioclimaticheskaya ziklichnost v Sankt-Peterburge v konze $\mathrm{XX}$ v. i ee vlijanie na introduzirovannuju i mestnuju dendrofloru. - Bot. Zhurn. 94: 1351-1358.

Fadeyeva, I.V. \& Firsov, G. A. 20io: Indikazionnoje znachenie dendrofenologicheskogo rjada zazvetanija Alnus incana $\mathrm{v}$ fenostazionare Sankt-Peterburgskoj lesotehnicheskoj akademii. Dendrologija v nachale XXI veka. Sb. mater. Mezhd. nauch chtenij pamjati E.L. Wolfa, 6-7 Oct. 2010, S. 210-214.

Firsov, G.A., Fadeyeva, I.V. \& Volchanskaya, A.V 2008: Vlijanie meteo-fenologicheskoj anomalii zimi 2006/07 goda na drevesnie rastenija v Sankt-Peterburge . - Vestnik-Lesnoj vestnik 6: 22-27.

Firsov, G. A., Fadeyeva, I.V. \& Volchanskaya A.V. 20I0: Fenologicheskoje sostojanie drevesnih rastenij v sadah i parkah S.-Peterburga v svjazi s izmenenijami

klimata. - Bot. Zhurn. 95: 23-37.

Shver Z.A., Altykis E.V. \& Evteyeva, L.S. (Hrsg.) 1982: Klimat Leningrada. - Leningrad.

Grimshaw, J. \& Bayton, R. 2009: New Trees: Recent introductions to cultivation. - Kew.

Heinze, W. \& Schreiber, D. I984: Eine neue Kartierung der Winterhärtezonen für Gehölze in Mitteleuropa. - Mitt. Deutsch. Dendro. Ges. 75: 11-56.

Komarova, V. N. \& Firsov, G. A. 1989: Reakzija drevesnih rastenij Sankt-Peterburga na meteoanomalii. Bull. Glav. Botan. Sada. Vip. 172: 8-10.

Pokrovskaya T.V. \& Bychkova A.T. 1967: Klimat Leningrada i ego okrestnostej. - Leningrad.

Shver Z.A., Altykis E.V. \& Evteeva, L. S. (Hrsg.) 1982: Klimat Leningrada. - Leningrad.

Wolf, E. L. 1917: Nablyudenija nad morozostojkostju derevjanictih rastenij. - Trudi Byuro po Prikl. Bot. 10: 1-146.

Wolf, E. L. 1929: Park i arboretum leningradskogo lesnogo instituta. - Mitt. Leningr. Forstinst. 37: 235-268.

Abb. 8 (oben): Gewürzstrauch (Calycanthus floridus).

Abb. 9 (Mitte): Dieser Ahorn (Acer mandshuricum) wurde noch von WoLf gepflanzt.

Abb. 10 (unten): Jungpflanzen eines japanischen Ahorns (Acer miyabei); die Samen stammen aus dem Arboretum in St. Petersburg. 